\title{
Técnicas participativas en el aprendizaje de las Ciencias Naturales.
}

\section{Participatory techniques in the learning of Natural Sciences.}

\author{
Mariela Cristina Lasluisa Caguana ${ }^{1}$, Raúl Yungán Yungán² ., María Cecilia Guayta \\ Sailema ${ }^{3}$ \& Silvia Beatriz Acosta Bones ${ }^{4}$.
}

\begin{abstract}
DOI: https://doi.org/10.33262/cienciadigital.v9i2.386

Participatory techniques are very important tools in education, which at present must be applied in all areas of studies. This article intends to investigate the incidence of participatory techniques in the learning of the Natural Sciences of the seventh grade students of Basic General Education of the Educational Unit "General Córdoba" of the canton Ambato, province of Tungurahua within the Ecuadorian area, for which the definition of the 2 variables was investigated. Importance in the field and educational context, besides in this work we propose participatory exercises in the learning of the natural sciences, designed under the principles of constructivism, transferring the protagonist of the teachers to the students. The article aims to investigate how the participatory techniques in the natural science learning of the seventh-grade general education students of the General Education Unit "General Córdoba" influence. The methodology used is the quantitative analysis with the structured survey technique in 2 blocks a. Training b. Research line to 5 teachers and 35 students of the General Cordova Educational Unit in the academic cycle September 2016-July 2017. The results are organized in two dimensions: (a) participatory techniques, (b) learning of the natural sciences. The result is a systematization that helps to understand the importance of participatory techniques in the learning process of the natural sciences.
\end{abstract}

Keywords: Participatory Techniques, Learning, Natural Sciences.

\footnotetext{
${ }^{1}$ Universidad Técnica de Ambato, Facultad de Ciencias Humanas y de la Educación, Carrera de Educación Básica, Ambato, Ecuador, maryuusp@gmail.com

${ }^{2}$ Universidad Técnica de Ambato, Facultad de Ciencias Humanas y de la Educación, Carrera de Educación Básica, Ambato, Ecuador, ryungan@uta.edu.ec

${ }^{3}$ Unidad Educativa Bolívar, Ambato Ecuador, maria.guayta@educacion.gob.ec

${ }^{4}$ Universidad Técnica de Amato, Facultad de Ciencias de la Educación, Carrera de Educación Inicial. Ambato, Ecuador. silviabacostab@uta.edu.ec
} 


\section{Resumen}

Las técnicas participativas son herramientas muy importantes en la educación, que en la actualidad deben ser aplicadas en todas las áreas de estudios. En el presente artículo se pretende investigar la incidencia de las técnicas participativas en el aprendizaje de las Ciencias Naturales de los estudiantes de séptimo Año de Educación General Básica de la Unidad Educativa "General Córdova" del cantón Ambato, provincia de Tungurahua, Ecuador para lo cual se indagará la definición de las 2 variables. Las técnicas participativas dentro del contexto educativo, en la cual proponemos ejercicios participativos en el aprendizaje de las ciencias naturales, diseñados bajo los principios del constructivismo, trasladando el protagonismo de los profesores a los estudiantes. El artículo tiene como objetivo investigar cómo inciden las técnicas participativas en el aprendizaje de las ciencias naturales de los estudiantes de séptimo grado de educación general básica de la Unidad Educativa "General Córdova". La metodología empleada es el análisis cuantitativo con la técnica la encuesta estructurada en 2 bloques a. lineamiento de formación b. lineamiento de investigación a 5 docentes y a 35 estudiantes de la Unidad Educativa "General Córdova", en el ciclo académico Septiembre 2016Julio 2017. Los resultados se organizan en dos dimensiones: (a) técnicas participativas, (b) aprendizaje de las ciencias naturales, el resultado es una sistematización que contribuye a comprender la importancia de las técnicas participativas en el proceso de aprendizaje de las ciencias naturales.

Palabras clave: Técnicas Participativas, Aprendizaje, Ciencias Naturales, Didáctica.

\section{Introducción.}

La técnica participativa es fundamental en la educación ya que fomenta, la creatividad. "Se originan de la práctica pedagógica, ya que es fundamental en proceso de enseñaaprendizaje en los principios de la educación. Se puede decir que son respuestas pedagógicas para resolver problemas del aprendizaje y más aún que es necesario en el área de ciencias naturales ya que son herramientas de apoyo" (Morales, 2015, p. 25). Ya que las estrategias didácticas de aprendizajes deben estar presentes en todo momento, para otorgarles una educación de calidad a los estudiantes.

En la actualidad la educación se ha ido transformando de mejor manera, debido a los cambios del entorno social, político, económico, cultural, científico y tecnológico. De acuerdo a los nuevos enfoques de la educación, es importante que la formación de los estudiantes en todos los niveles se fortalezca con los conocimientos científicos de tal manera que le permitan a cada individuo una visión crítica del mundo desde el plano investigativo. Se evidencia una mejora significativa en el sistema educativo del Ecuador, destacando la área de Ciencias Naturales así superando a 9 países, de esta manera podemos llegar a los primeros lugares si todos los docentes aplicaran las técnicas participativas en las aulas de clases. ( Ministerio de Educación 2011, pág. 4) 
Cabe decir que, destaca el ministerio de educación en buscar mejoramiento de la metodología de los docentes, al momento de operatividad en las aulas de clases de esta manera construir un aprendizaje significativo. Donde el estudiante sea el actor principal en la construcción de sus pensamientos y aprendizajes nuevos. Ya que en el área de Ciencias Naturales es primordial trabajar con actividades participativas que pueda despertar interés e indagación de nuevas teorías.

"Es indispensable enfocar que las técnicas participativas es parte de una metodología ampliamente que se debe utilizar en todas las aulas de clases, ya que son instrumentos que orienta al estudiante a una activa y participativa intervención con los docentes y es de gran ayuda al proceso de enseñanza-aprendizaje" (Alvarez, Silegas, \& Rosales, 2002, p. 34).

Cabe decir que, los autores enfatizan que las técnicas participativas, son instrumentos muy necesarios dentro del proceso aprendizaje significativo de los estudiantes.

El aprendizaje de las Ciencias Naturales, se puede decir que tradicionalmente se reduce a las clases expositivas por el docente, a estudiar de manera significativa de memoria los contenidos de los libros del ministerio de educación y en algunas ocasiones, a demostraciones para comprobar algún hecho.

Se debe tomar en cuenta que estas actividades no garantizan el desarrollo de las habilidades y destrezas para la investigación, que es el objetivo fundamental para la mejor comprensión de las Ciencias Naturales. El lineamiento de la didáctica de las Ciencias Naturales enfoca en ofrecer elementos y cursos metodológicos para favorecer una mentalidad científica y actitud dinámica y participativa en la dirección del curso de las Ciencias Naturales. En los estudiantes se busca sensibilizar la importancia del papel y la orientación que deben tener en el área de las Ciencias Naturales, fortaleciendo así la Educación Científica, de esta manera se podría mejorar los aspectos más prácticos y operativos para facilitar los procesos de aprendizaje relacionados al área de estudio. (Roncal \& Federico, 2000, p. 28)

De la misma manera, los autores argumentan el objetivo fundamental para un buen aprendizaje de las Ciencias Naturales, es enfocar nuevos elementos y cursos metodológicos donde participen activamente todos los estudiantes. Para favorecer una mentalidad científica y actitud dinámica en la construcción de los nuevos aprendizajes.

La sociedad va cambiando mediante algunos procesos sistemáticos de transformación en los que indispensablemente participan individuos como sujetos que investigan y evalúan por medio de acciones colectivas, las cuales analiza las tres clases de conocimientos, creativo, interactivo y crítico a fin que es necesario reexaminar la base metodológica de las Ciencias Naturales convencionales en la búsqueda de un nuevo paradigma más acorde con los rasgos distintivos de la sociedad humana en contraposición al mundo natural. Analiza la participación y compromiso del investigador, los procesos de la investigación participativa en todos deben cumplir su rol para una determinada actividad colectiva. 
Cabe destacar que la metodología participativa lleva a que las personas fomenten la capacidad de pensar por sí misma, de innovar y mediante la reconstrucción de su historia y su entorno natural para lograr una vida autentica. (Kurt, Sol, Stavenhagen, \& Kemmis, 2001, p. 8)

De hecho, donde los autores especifican que de acuerdo a los años la sociedad va cambiando mediante algunos procesos sistemáticos de transformación en los que indispensablemente son necesarios que participan individuos que investiguen y evalúen acciones participativas, para colaborar en la construcción de nuevos aprendizajes.

\section{Técnicas Participativas.}

Las técnicas participativas de hecho, no pasan de ser herramientas, dentro de un proceso que ayuda a fortalecer la organización, participación y concentración. Según Martin, (2005) "son los recursos y procedimientos que abarca en una metodología dialéctica permiten repensar la práctica de los participantes, para fortalecer el desarrollo científico para beneficiar a todas las personas, principalmente la educación, todo el conocimiento necesario e indispensable para transformar y recrear nuevas prácticas” (p. 8).

Desarrollar un proceso sistemático, donde siempre se debe enfocar en la práctica, o sea partir de lo que la gente sabe, vive y siente; las diferentes situaciones y problemas que enfrentan en su vida diaria, y que en un programa educativo se plantean actividades a desarrollar. Los métodos participativos abarcan todas las técnicas que faciliten la participación democrática de todo el conjunto de personas en el proceso de reflexión y análisis. Con una buena aplicación de las técnicas participativas facilitan el rompimiento de la dinámica formal que en la mayoría afecta en la mayoría de personas. Para así fortalecer el fluido libre de ideas y opiniones. (Reflect, 2009, p. 12)

En lo manifestado, se debe enfocar en la práctica, o sea a partir de los conocimientos adquiridos, en el diario vivir de acuerdo a las diferentes situaciones y problemas que se presentan diariamente. Ya que en un programa educativo se plantea desarrollar actividades, con métodos participativos, donde la creatividad e imaginación de cada participante facilite una participación democrática.

Las metodologías pedagógicas que proponemos están ligadas a un uso no tradicional; son los espacios participativos de los estudiantes y de la pedagogía universitaria, implícita en el proceso de enseñanza-aprendizaje. Procuramos una metodología eficaz para la enseñanza de los conocimientos y el desarrollo de habilidades esenciales propias del quehacer de las ciencias naturales. Estas modalidades de enseñanza participativa han sido utilizadas en la enseñanza de las ciencias naturales en distintos sistemas académicos, e incluyen: a) estudios de casos (de variada naturaleza), b) talleres, c) discusiones lideradas por los estudiantes, d) proyectos de campo basados en estudios de historia natural, e) prácticas de laboratorios y parcelas en el campo, orientados por el método científico, y f) coloquios. (Gutiérrez, Arias, \& Luis, 2009, p. 15-20) 
La metodología de trabajo en la gestión participativa de los procesos de desarrollo, se fundamenta en los principios pedagógicos del "aprender haciendo" y avanza "de lo sencillo al complejo", aplicando cabalmente el concepto de la reflexión-acción o teoríapraxis. Con la investigación participativa se logra colectivamente describir, analizar y priorizar las limitaciones, necesidades, problemas y potencialidades de un espacio y un territorio concreto.

"La socialización del saber local se propicia una comunicación intergeneracional interna y una comunicación interactiva entre los actores locales y los promotores externos. Con la planificación participativa se aprende mientras se formula y ejecuta ese plan. Se diseñan el horizonte, los objetivos y los indicadores que luego permiten realizar el seguimiento, control y evaluación de las acciones ejecutadas en el planeamiento estratégico y operativo" (Cox, 1996, p. 27).

La participación grupal permite desarrollar una experiencia de reflexión educativa común. Muchas de estas técnicas permiten tener un punto común de referencia a través del cual los participantes aportan su experiencia particular, enriqueciendo y ampliando de esa experiencia colectiva.

Las técnicas Participativas constituyen herramientas, instrumentos útiles para las estrategias de intervención en grupos colectivos. Participar en un grupo significa indagar un cambio en el proceso que se ha establecido, para los integrantes del grupo. El cambio debe ser sistematizado, éticamente correcto y requiere de una tecnología específica y de una concepción teórica metodológica. Además, estas técnicas permiten crear un espacio y establecer una relación mejor entre docente y alumno. Cabe recalcar que solo son instrumentos en proceso de formación, se caracterizan por su carácter abierto, flexible, participativo grupal y práctico vivencial. Favorecen la participación y la implicación individual y colectiva, de manera que los contenidos del proceso educativo adquieran un sentido personal para los participantes y la habilidad de trabajar en grupo colectivamente. (Rodriguez \& Oramas, 2002, p. 45)

Cabe decir que, se argumenta que las técnicas participativas, son instrumentos muy necesarios e importantes dentro de las estrategias de intervención en grupos colectivos. Ya que una participación es necesario indagar e investigar para poder construir nuevos conceptos e ideas dentro del grupo, ya que estas técnicas permiten crear un espacio y establecer una relación mejor entre docente y alumno.

Olla de Sueños Objetivos: Conocer la visión de las personas participantes, sobre un mundo más justo y humano. (La aplicacion del enfoque basado en derechos., 2009, pág. 59)

Monitoreo Participativo: Elaborar en forma rápida y libre una lista de asuntos, temas y preguntas para el monitoreo de un proyecto, haciendo uso de las percepciones colectivas del grupo. (La aplicacion del enfoque basado en derechos., 2009, pág. 62) 
Árbol de problemas: El Árbol de Problemas nos sirve para identificar los síntomas que dan cuenta de un problema, y para relacionar estos con el análisis de sus causas inmediatas y sus causas profundas. Las posturas a debatir para colocar en el árbol se pueden traer de las frases ya recogidas en el trabajo de campo anterior, y se pueden completar también con las aportaciones de quienes acudan a sesiones de trabajos en grupos y plenario. (Tecnicas Participativas, pág. 45)

Socio-drama El socio-drama es un método según el cual un grupo estudia un tema en concreto, una situación social o a sí mismo mediante un proceso de grupo creativo guiado por un instructor. Son los mismos miembros del grupo quienes pueden escoger los roles o bien se les pueden ser asignados. (Tecnicas Participativas, p. 48)

\section{Aprendizaje de las Ciencias Naturales}

La tarea de aprender ciencias y en particular ciencias naturales, se destaca en la actualidad, el desafío de las nuevas alfabetizaciones. Consideramos que aprender ciencias permite adquirir conocimientos sobre las bases teóricas en que se fundamenta la didáctica de las ciencias experimentales. Según Riveros, (2011) afirma "que los docentes deben saber que hay más de una forma de explicar que es la ciencia, los contenidos para aprender en el aula. Además, es importante reflexionar acerca como aprenden los estudiantes y a investigar las teorías actuales sobre el aprendizaje, en particular las propuestas desde el campo de la didáctica de las ciencias para interpretar las dificultades de los alumnos en su aprendizaje, también influyen los factores personales y sociales para su aprendizaje" (p. 28).

Según Chamorro, (2013) afirma "En las instituciones educativas un objeto fundamental debe orientarse a proveer el dominio de las herramientas para mejorar el aprendizaje de las Ciencias Naturales, que les permitan a los individuos tanto apropiarse del conocimiento y ponerlo en práctico como construirse a sí mismos y a una sociedad más justa” (pág. 10).

La colección Teoría y práctica curricular de la educación básica se suma a otras accione de producción de materiales y desarrollo de actividades de actualización que mejore el aprendizaje y la comprensión de las ciencias naturales. "En cada volumen se presenta un panorama actualizado del desarrollo de las didácticas de las asignaturas, así como son sus enfoques pedagógicos y las sugerencias para su tratamiento en cada nivel educativo" (Gobierno Federal, 2011, p. 36).

Ciencia- Educación en la actualidad es un tema muy transversal en el sistema educativo, que nace desde una alianza. En ella, científicos y educadores nos proponen una metodología del aprendizaje de las Ciencias Naturales basada en la indagación, que permite a los niños y niñas aprender ciencias desde muy temprana edad, convirtiéndoles en principales protagonistas de experiencias adecuadas y significativas que facilitan aprender no solo los contenidos sino, además, los procesos que permiten aceptarlos como correctos y verdaderos, es importante destacar que esta tendencia mundial en el 
aprendizaje de las ciencias basada en la indagación, es desarrollada y propuesta desde una necesidad de que los niños y niñas aprenden habilidades y destrezas que provengan del mundo de las ciencias pero que pueden ser aplicadas en el diario vivir. ( Educarchile, 2010, p. 57)

En consecuencia, se puede afirmar que la Ciencia en la Educación en la actualidad es muy tema muy transversal e importante dentro del sistema educativo, donde algunos científicos y educadores nos proponen una metodología del aprendizaje de las Ciencias Naturales, se basen más en la indagación que permita a los niños y niñas a ser los protagonistas que faciliten el proceso de aprendizaje significativo.

\section{Estrategia didáctica}

Las actividades académicas deben cumplir con un conjunto de procedimientos, pasos y ciertas actividades que permitan a los y las estudiantes acceder al conocimiento y a los saberes de una manera activa, participativa y significativa; teniendo como sustento que toda actividad educativa deben cumplirse un ciclo ordenado para lograr los objetivos propuestos.

En consecuencia, la planificación de clase debe tomar en cuenta pasos secuenciales para cada una de las técnicas, de manera que, tales pasos se convierten en una camisa de fuerza, por el contrario, cada docente podrá aplicar desde su experiencia aplicando métodos de la manera más conveniente y el momento más oportuno.

Con el fin de mejorar la educación el Ministerio de Educación, (2012) diseña y publica el texto Didáctica de la enseñanza de la Ciencias Naturales y en como objetivos del curso plantea los siguientes objetivos.

- Revisar las concepciones sobre el para qué y cómo del aprendizaje de Ciencias Naturales.

- Guiar a sus estudiantes a comprender la complejidad de la interacción de los diversos elementos de la naturaleza (incluyendo el ser humano), para explicar las diversas formas de vida en la Tierra y el impacto de las acciones humanas en su destrucción o preservación.

- Conducir el aprendizaje de Ciencias Naturales desde y para la resolución de problemas reales.

- Promover el aprendizaje de Ciencias Naturales desde los desempeños propios de los científicos.

- Guiar en sus estudiantes la integración de saberes de las distintas ramas de las Ciencias Naturales, a partir del análisis y presentación de soluciones ante problemas socio-ambientales.

- Identificar sus fortalezas y debilidades en el manejo de conceptos de las varias disciplinas que componen el campo de las Ciencias Naturales y emprender planes personales de profundización disciplinar. (Ministerio de Educación, 2012, p. 8) 
Estos objetivos permiten a que los maestros y maestras estén capacitados y actualizados en la operativización de la asignatura de las Ciencias Naturales. Así como el desarrollo de los contenidos permitan la integración de saberes de las distintas ramas de ciencias naturales y otras ciencias con el fin de analizar y buscar las soluciones inmediatas.

En este mismo sentido el Ministerio de Educación, ( 2014) diseña y publica el texto Guía para Docente de Ciencias Naturales, esta guía constituye en una herramienta de apoyo para los maestros y maestras y pretende entre sus objetivos principales de dotar al maestro de un documento práctico que permita direccionar la labor docente en cada uno de los bloques curriculares.

Con facilidad y en todos los medios se puede encontrar productos y actividades para niños y niñas, donde aparece el concepto de didáctica, contenidos didácticos, material didáctico y juegos didácticos, sin embargo, muchas veces perdemos de vista las definiciones teóricas y nos quedamos sin profundizar, por esta razón es interesante que la institución rectora otorgue guías a los docentes, de manera que la técnicas y métodos serán de prioridad en el proceso de aprendizaje.

\section{Metodología.}

Para la elaboración del trabajo de investigación se tomó como base el paradigma critico propositivo pues a partir del problema se plantea la incidencia de la variable independiente sobre la dependiente en este caso se analizará las técnicas participativas y su influencia en el aprendizaje de las ciencias naturales para lo cual se realizó encuestas dirigidas a estudiantes y docentes de los séptimos años de Educación General Básica que dan en una totalidad de 40 personas distribuidos en 35 estudiantes y 5 docentes pertenecientes a la Unidad Educativa "General Córdova".

Después de haber ejecutado la encuesta a la población educativa se tabulo y se analizó la influencia de la variable principal (Técnicas Participativas) sobre la secundaria (Aprendizaje de las Ciencias Naturales) a través de un instrumento estadístico conocido como CHI cuadrado que brinda una mayor exactitud en el cálculo respectivo " Para determinar la asociación o independencia de dos variables cualitativas con un cierto grado de significancia, se dispone de una herramienta estadística frecuentemente utilizada, el test de chi-cuadrado (X2), afirmación que cuenta con un sustento estadístico" (Cerda \& Villaroel, 2007, págs. 6-7). Obteniendo como resultado que se debe aplicar frecuentemente las diferentes técnicas participativas en las aulas de clases de esta manera desarrollar el aprendizaje de las ciencias naturales en los estudiantes conjuntamente con el apoyo de las demás herramientas didácticas empleadas por el docente, que faciliten el proceso de formación integral de todos los estudiantes.

En esta herramienta de cálculo se empieza elaborando las tablas de frecuencias observadas $(\mathrm{O})$ seguidamente las tablas de frecuencias esperadas $(\mathrm{E})$ de esta manera se ejecutan el cálculo Chi cuadrado $\left(\mathrm{x}^{2}\right)$ en base a lo anterior, para lo cual se utiliza la siguiente descripción: 


$$
x^{2}=\frac{\sum(O-E)^{2}}{E}
$$

En este estadístico se trabajó con seis grados de libertad con un nivel de significación de 0,05 y en un margen de error de 0,0. A continuación se presenta la descripción sobre la muestra poblacional con la cual se trabajó para la elaboración del presente documento.

Tabla 1: Muestra Poblacional

\begin{tabular}{|lcccc|}
\hline \multicolumn{5}{|c|}{ MUESTRA POBLACIONAL } \\
\hline Año lectivo & Estudiantes & Docentes & Total & Porcentaje \\
\hline 2016- 2017 & \multicolumn{4}{c|}{ Séptimo año } \\
\hline & 35 & 5 & 40 & $100 \%$ \\
\hline
\end{tabular}

\section{Elaborado: Autores}

Se estableció el contacto respectivo con la población a quien se aplicó el instrumento para la realización de esta investigación pidiendo su anticipado conocimiento por parte de la Institución educativa y los principales beneficiarios siendo en este caso estudiantes y docentes para lo consiguiente en este proyecto, la herramienta empleada fue una encuesta formada por 10 preguntas dirigida tanto a estudiantes y a docentes en horario escolar lo cual nos permitió realizar las tabulaciones y sus respectivos análisis e interpretaciones a partir de su cálculo independiente es decir pregunta por pregunta en una hoja de Microsoft Excel especificando sus diferentes alternativas.

\section{Resultados.}

Las técnicas se usan para que los estudiantes participen, se animen y se integren juntos en la construyan el conocimiento entre todos y todas, también deben permitir en hacer más sencillo y comprensible los temas y contenidos de las diferentes áreas. Por tanto, se procede a indagar el uso de las técnicas. De acuerdo a la información receptada de la pregunta ¿El docente utiliza técnicas participativas en el desarrollo de la clase? se obtiene la la siguiente información.

De 35 estudiantes que corresponden al 100\%, 20, el 57\% dicen que a veces los docentes utilizan técnicas participativas en el desarrollo de la clase mientras que el $37 \%$ manifiestan que siempre y el 6\% indican que nunca. Es decir, desde la percepción de los estudiantes los maestros y las maestras en su gran mayoría no utilizan las técnicas participativas. Las Técnicas dinámicas o vivenciales; que se trata de la práctica diaria. Dentro de esta técnica encontramos las de animación y de análisis que es muy factible realizarlas en las aulas de clase. También hay la técnica de actuación que es imprescindible aplicar ya que se trata de la expresión corporal donde se refleja el comportamiento de cada individuo. 
Técnicas auditivas y visuales que son imágenes, videos, power point que ayuda para una mejor comprensión de las ciencias naturales en los estudiantes. De esta manera se puede visualizar que hay un porcentaje alto de estudiantes que coinciden que a veces el docente utiliza técnicas participativas en el desarrollo de la clase, esto dificulta el aprendizaje de los estudiantes.

En este mismo sentido se plantea la interrogante $¿ E l$ docente permite realizar actividades participativas fuera del aula, para el aprendizaje de las Ciencias Naturales?, al respecto se obtiene la siguiente información.

De 35 estudiantes que corresponden al 100\%, el 46\% indican que nunca el docente permite realizar actividades participativas fuera del aula, para el aprendizaje de las Ciencias Naturales, mientras que el $43 \%$ manifiestan que a veces y el $11 \%$ indican que siempre. De acuerdo a los resultados se visualiza que el docente no utiliza técnicas participativas ni creativas fuera del aula de clase.

Existen muchas técnicas visuales que el maestro y la maestra puede dar uso en el proceso de aprendizaje, donde el estudiante pueda expresar libremente lo observado. Además, el trabajo al aire libre permite colectivizar ideas, resumir o sintetizar discusiones ya que favorecen la participación de todos los estudiantes.

En consecuencia, de los resultados obtenidos se propone y sugiere dos técnicas participativas y característica que debe tener un/a Educador/a en Ciencias Naturales.

\section{Técnica participativa 1}

Tema: "Quién soy"

Contenido: Biodiversidad

Objetivo: Realizar una caracterización rápida de algún animal doméstico.

\section{Actividades:}

1. Los estudiantes se ubican en círculo y el docente solicita un voluntario o voluntaria.

2. Sin que el estudiante voluntario lo vea, se le adhiere en la espalda un letrero con el nombre de un animal doméstico que representa.

3. El voluntario va preguntando a los demás estudiantes acerca de sus características.

4. Los participantes del circulo sólo pueden responder SI o NO, cuando el voluntario adivina que animal es, se cambia de participante. 


\section{Sugerencias}

Es posible ampliar con otros nombres de los animales.

Hacer las actividades en el patio o un bosque cercano.

\section{Técnica participativa 2}

Tema: "Escuche un mensaje"

Contenido: Ecosistema - Biodiversidad

Objetivo: Comparar las características de los diferentes componentes de algún ecosistema elegido.

\section{Actividades:}

1. Cada participante se convierte en un elemento del ecosistema y debe pegarse en el pecho un papel con un nombre de un animal, ejemplo: (llama, venado, borrego, caballo, lobo, cóndor, gavilán, entre otros), que el docente repartiera.

2. Formar un circulo y el docente de pie iniciará el juego diciendo "escuche un mensaje”, al cual los estudiantes responderán “¿para quién?”. El docente señalará alguna característica común para varios estudiantes, por ejemplo "para los que vuelan" o "para los que tienen plumas" o para los que comen granos. Aquí depende de la habilidad del docente.

3. Todos los estudiantes que cumplan con las características mencionadas deberán cambiar de asiento y el docente ocupará alguno disponible.

4. El o la estudiante que quede parado que no cambien de asiento o se equivoque, debe ponerse de pie en el centro del circulo y decir "escuche un mensaje".

5. Así sucesivamente puede continuar la técnica participativa.

\section{Sugerencias}

$>$ Esta actividad puede aumentar la complejidad

Utilizar en otros temas

Los errores contribuyen a la profundización del tema

\section{Técnica participativa 3}

Tema: "Láminas y Adivinanzas"

Contenido: Ecosistema 
Objetivo: Expresar las peculiaridades y necesidades vitales de los seres vivos.

\section{Actividades:}

Antes de comenzar la actividad preparar láminas de elementos vivos e inertes (dibujar o recortar de revistas, periódicos).

1. Combine las láminas y forme una ronda con los participantes sentados en el piso.

2. Intercambie las figuras, reservando una para el educador.

3. Las ilustraciones de las láminas se mantendrán en secreto.

4. Para empezar con el juego, el docente mira su lámina y utiliza su imaginación para crear una adivinanza sobre la imagen. Por ejemplo, habla y no tiene boca, oye y no tiene oído, es chiquito y hace ruido, muchas veces se equívoca. ¿Quién soy? El teléfono. Es venta y no vende, es Ana pero no es gente. ¿Quién soy? La ventana.

5. Cuando los participantes resuelvan la adivinanza, el docente gira la lámina para que todos alcancen a mirar, después de dar los ejemplos se invita a todos a participar en la ronda de la misma manera que explico anteriormente en el ejemplo realizado.

6. A continuación, se les propone que realicen una clasificación de las figuras con las que estaba trabajando, es muy posible que surjan inconvenientes hasta llegar a clasificar seres vivos e inertes. Pero de esta manera se genera pensamiento crítico e intercambio de ideas.

7. Luego se dibujan dos grandes bolsas en un papelógrafo o en el pizarrón. Una bolsa será para los elementos vivos y la otra para los inertes. Los participantes irán pegando sus láminas en la bolsa que corresponda.

\section{Sugerencias}

$>$ Esta actividad se puede utilizar con otros materiales didácticos.

Utilizar en temas complejos.

(estas actividades fueron tomada y modificada del texto Martínez, J. (ed). Dinámicas grupales, Chile 1991)

\section{Características del Educador/a}

Persona capaz de diagnosticar las condiciones y demandas del entorno natural.

$>$ Contar con una solidad formación en las ciencias Naturales.

Manejar las técnicas participativas dentro y fuera del aula. 
Desarrollo de aptitudes y estrategias cognitivas que permitan desenvolverse eficiente y eficazmente frente a los estudiantes.

> Capacidad de criticidad, comprendida con el desarrollo de criterios para enjuiciar, evaluar y proponer.

\section{Conclusiones.}

- Las técnicas participativas en la asignatura de Ciencias Naturales, son consideradas como un componente de la metodología, como el medio o procedimiento que se utiliza para la construcción del conocimiento, con la participación de los actores educativos, creando de esta manera un ambiente fraterno, participativo entre los actores educativos.

- Se mejoró la calidad de la educación al aplicar algunos de los ejercicios propuestos, que fue una estrategia para que los maestros de Ciencias Naturales, realizaran técnicas participativas que contribuyen al desarrollo integral de los alumnos y alumnas. Cabe recalcar que la aplicación de técnicas didácticas mejora significativamente el aprendizaje de los estudiantes ya que las técnicas participativas contribuyen con el aprendizaje de Ciencias Naturales de gran manera, lo mejoran, su aplicación es provechosa.

- La identificación de los métodos y técnicas grupales como alternativa promisoria para lograr el incremento del nivel de asimilación de los contenidos de Ciencias Naturales por parte de los estudiantes es fundamental ya que la introducción de indicaciones y normas de carácter general que faciliten la implementación de las técnicas grupales en la práctica docente.

- El tipo de técnicas participativas de aprendizaje que utilizan los docentes son aquellas que fomentan el trabajo en equipo, exposiciones, utilizadas solo algunas veces en el aula de clases, otras como dinámicas activas y vivenciales, juegos populares, sociodramas son desarrolladas de manera muy esporádica por lo cual, la ejemplificación de cómo pueden aplicarse algunas técnicas grupales en el proceso de aprendizaje de los contenidos de Ciencias Naturales.

\section{Referencias Bibliográficas}

Educarchile. (17 de Diciembre de 2010). Metodología indagatoria para aprender ciencias. Recuperado el 21 de Julio de 2017, de http://m.educarchile.cl/portal/mobile/articulo.xhtml?id=186632

Ministerio de Educación 2011. (s.f.). Ecuador mejoró su sistema educativo en los últimos 7 años. Recuperado el 14 de Julio de 2017, de https://educacion.gob.ec/ecuadormejoro-su-sistema-educativo-en-los-ultimos-7-anos/

Aldana, S., Vereda, F., Hidalgo-Alvarez, R., \& de Vicente, J. (2016). Facile synthesis of 
magnetic agarose microfibers by directed selfassembly. Polymer, 93, 61-64.

Alvarez, S., Silegas, M., \& Rosales, J. (2002). Técnicas participativas y su uso en la enseñanza- aprendizaje. MEDISAN, 20-25.

Bhat, S., Tripathi, A., \& Kumar, A. (2010). Supermacroprous chitosan-agarose-gelatin cryogels. in vitro characterization and in vivo assesment for cartilage tissue engineering. Journal of the Royal Society Interface, 1-15.

Bossis, G., Marins, J., Kuzhir, P., Volkova, O., \& Zubarev, A. (2015). Functionalized microfibers for field-responsive materials and biological applications. Journal of Intelligent Material Systems and Structures, 1-9.

Cerda, J., \& Villaroel, L. (2007). Interpretación del test de Chi-cuadrado (X2) en investigación pediátrica. Revista chilena de pediatría, 6 .

Chamorro, D. (2013). El lenguaje para enseñar y aprender las Ciencias Naturales. Revista signos, 23.

Cortés, J., Puig, J., Morales , J., \& Mendizábal, E. (2011). Hidrogeles nanoestructurados termosensibles sintetizados mediante polimerización en microemulsión inversa. Revista Mexicana de Ingeniería Química., 10(3), 513-520.

Cox, R. (1996). El Saber Local: Metodologías y Técnicas Participativas. Obtenido de http://www.cedet.edu.ar/Archivos/Bibliotecas_Archivos/El\%20saber\%20local.p df

Dias, A., Hussain, A., Marcos, A., \& Roque, A. (2011). A biotechnological perspective on the application of iron oxide magnetic colloids modified with polysaccharides. Biotechnology Advances 29 , 29, 142-155.

Estrada Guerrero, R., Lemus Torres, D., Mendoza Anaya, D., \& Rodriguez Lugo, V. (2010). Hidrogeles poliméricos potencialmente aplicables en Agricultura. Revista Iberoamericana de Polímeros, 12(2), 76-87.

García-Cerda, L., Rodríguez-Fernández, O., Betancourt-Galindo, R., Saldívar-Guerrero, R., \& Torres-Torres, M. (2003). Síntesis y propiedades de ferrofluidos de magnetita. Superficies y Vacío., 16(1), 28-31.

Gobierno Federal. (2011). Teoría y Práctica Curricular de la Educacion Básica. Mexico: Cuauhtémoc.

Gutiérrez, M., Arias, J., \& Luis, P. (2009). ESTRATEGIAS PARTICIPATIVAS PARA LA ENSEÑANZA DE LAS CIENCIAS NATURALES EN LA UNIVERSIDAD DE COSTA RICA. “Actualidades Investigativas en Educación”, 4-5.

Ilg, P. (2013). Stimuli-responsive hydrogels cross-linked by magnetic nanoparticles. Soft 
Matter, 9, 3465-3468.

Investigación e Innovación Educativa. (2010). Técnicas Didácticas. Recuperado el 28 de Julio de 2017 , de http://sitios.itesm.mx/va/dide2/tecnicas_didacticas/quesontd.htm

Kurt, L., Sol, T., Stavenhagen, R., \& Kemmis, S. (2001). La Investigación Acción Participativa. Colombia: O.E.I. Quinto Centenario.

Lewitus, D., Branch, J., Smith, K., Callegari, G., Kohn, J., \& Neimark, A. (2011). Biohybrid carbon nanotube/agarose fibers for neural tissue engineering. Advanced Functional Materials, 21, 2624-2632.

Lin, Y.-S., Huang, K.-S., Yang, C.-H., Wang, C.-Y., Yang, Y.-S., Hsu, H.-C., . . Tsai, C.-W. (2012). Microfluidic synthesis of microfibers for magnetic-responsive controlled drug release and cell culture. PLoS ONE, 7(3), 1-8.

Martin, E. (2005). Tecnicas Particpativas. Recuperado el 21 de Julio de 2017, de http://karin.fq.uh.cu/ vladimar/cursos/\%23Did\%E1cticarrrr/Libros\%20de\%20T emas\%20Pedag\%F3gicos/TecnicasParticipativasI.pdf

Mora, A. (2004). La Evaluación Educativa. Actualidades Investigativas en Educación, 2. Morales, A. (04 de Noviembre de 2015). Folleto de Técnicas Participativas . Recuperado el 28 de Julio de 2017, de http://www.efdeportes.com/efd211/folleto-de-tecnicasparticipativas-para-fisica.htm

Ortiz, B. (2002). Necesidad de Aplicación de Técnicas Participativas. Recuperado el 29 de Julio de 2017, de http://biblioteca.usac.edu.gt/tesis/07/07_1585.pdf

Reflect. (2009). Herramientas Participativas. Interesting Informative, 4.

Riveros, V. (2011). Por qué Aprender o Enseñar CIencias Naturales. Encuentro Educacional, 305.

Rodriguez, R., \& Oramas, A. (2002). Los Métodos Participativos en Pedagogía. Salud de los Trabajadores , 118.

Roncal, C., \& Federico, F. (2000). Didáctica de las Ciencias Naturales. Guatemala: EDUMAYA.

Ruiz Estrada, G. (2004). Desarrollo de un Sistema de liberación de fármacos basado en nanopartículas magnéticas recubiertas con Polietilénglicol para el tratamiento de diferentes enfermedades. Madrid: Universidad Autónoma de Madrid. Departamento de Física Aplicada.

Song, J., King, S., Yoon, S., Cho, D., \& Jeong, Y. (2014). Enhanced spinnability of narbon nanotube fibers by surfactant addition. Fiberes and Polymers, 15(4), 762- 
766.

Tartaj, P., Morales, M., González-Carreño, T., Veintemillas-Verdaguer, S., \& Serna, C. (2005). Advances in magnetic nanoparticles for biotechnology applications. Journal of Magnetism and Magnetic Materials, 290, 28-34.

Universidad de Chile. (2002). Técnicas Educativas. Recuperado el 28 de Julio de 2017, de file:///C:/Users/hp/Documents/Downloads/Tecnicas_educativas_I.pdf

Wulff-Pérez, M., Martín-Rodriguez, A., Gálvez-Ruiz, M., \& de Vicente, J. ( 2013 ). The effect of polymer surfactant on the rheological properties of nanoemulsions. Colloid and Polymer Science, 291, 709-716.

Zamora Mora, V., Soares, P., Echeverria, C., Hernández , R., \& Mijangos, C. (2015). Composite chitosan/Agarose ferrogels for potential applications in magnetic hyperethermia. Gels., 1, 69-80.

Zárate, H., \& Perez, M. (2004). El grupo en el aprendizaje. Recuperado el 28 de Julio de 2017, de http://www.unne.edu.ar/unnevieja/Web/cyt/com2004/1-Sociales/S039.pdf 


\section{Para citar el artículo indexado.}

Lasluisa M., Yungán R., Guayta M. \& Acosta S. (2019). Técnicas participativas en el aprendizaje de las Ciencias Naturales. Revista electrónica Ciencia Digital 3(2), 218-235. Recuperado desde: http://cienciadigital.org/revistacienciadigital2/index.php/CienciaDigital/article/view/386 1834

\section{\Ciencia}

El artículo que se publica es de exclusiva responsabilidad de los autores y no necesariamente reflejan el pensamiento de la Revista Ciencia Digital.

El artículo queda en propiedad de la revista y, por tanto, su publicación parcial y/o total en otro medio tiene que ser autorizado por el director de la Revista Ciencia Digital.
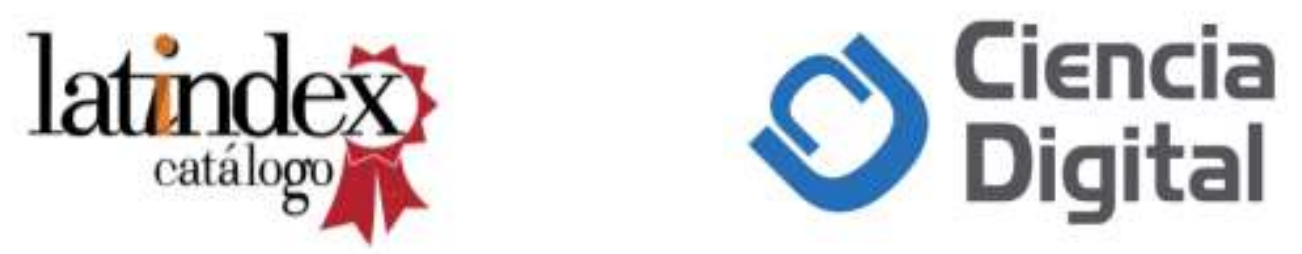